\title{
PERFIL SENSORIAL DO TOMATE DE MESA (Lycopersicon esculentum Mill.) ORGÂNICO
}

\section{SENSORY PROFILE OF ORGANIC TOMATO (Lycopersicon esculentum Mill.)}

\author{
FERREIRA, Sila Mary Rodrigues',2; FREITAS, Renato João Sossela de²; LAZZARI, Elisa \\ Noemberg3; QUADROS, Diomar Augusto de ${ }^{1,4}$
}

\author{
'Departamento de Nutrição da Universidade Federal do Paraná - Av. Lothário Meissner 3400, Campus III - Jardim Botânico Curitiba , PR, CEP: \\ 80210-170. Endereço eletrônico: sila@milenio.com.br. (autor responsável). \\ ${ }_{2}^{2}$ Programa de Pós-Graduação em Tecnologia de Alimentos - UFPR. \\ ${ }^{3}$ Bolsista PIBIC/UFPR e acadêmica do Curso de Nutrição da UFPR \\ ${ }^{4}$ Faculdades Integradas "Espírita". Curso de Nutrição \\ Recebido em:02/2004 \\ Aprovado em: 04/2004
}

RESUMO

Foram avaliadas, pela análise descritiva quantitativa - ADQ, quatro amostras de tomate de mesa, Lycopersicon esculentum Mill., cultivadas pelo sistema orgânico e comercializadas de dezembro de 2001 a fevereiro de 2002. A comparação realizada pelo teste de Tukey revelou que nos descritores defeitos de superfície, cor, firmeza ao toque, firmeza ao corte, resistência ao corte, aparência geral externa, espessura da polpa, sabor estranho, textura oral, doçura e acidez as amostras apresentaram diferença em nível de 5\% de probabilidade de erro. Nos atributos grau de suculência, característica da polpa, aroma, sabor, sabor remanescente, aparência geral interna e qualidade global as amostras não diferiram entre si.

Palavras-chaves: tomate orgânico, análise descritiva quantitativa - ADQ, análise sensorial.

ABSTRACT

Using Quantitative Descriptive Analysis (QDA), four samples of salad tomatoes (Lycopersicon esculentum Mill) were evaluated. The samples were organic grown tomatoes, commercialized from December 2001 to February 2002. Comparison using Tukey 5\% revealed significant difference concerning the following descriptors: surface defects, color, firmness to touch, firmness to cut, resistance to cut, overall exterior appearance, pulp thickness, strange taste, oral texture, sweetness and acidity. The descriptors juiciness, pulp characteristic, smell, taste, after taste, overall interior appearance and overall quality showed no significant difference.

Keywords: organic tomato, quantitative descriptive analysis - QDA, sensory analysis.

\section{INTRODUÇÃO}

As propriedades sensoriais do tomate de mesa são importantes tanto para a avaliação da qualidade do vegetal pelos consumidores como para procedimento da compra. No supermercado, somente aqueles produtos que correspondem as expectativas do consumidor são comercializados. Entretanto, é necessário identificar os fatores que influenciam a qualidade dos frutos e seus efeitos sobre as características sensoriais durante o período de pós-colheita.

O estádio de maturação do tomate influencia na vida pós-coclheita e no processo de amadurecimento, que por sua vez interfere diretamente na qualidade do produto que chega ao consumidor (MOURA; SARGENT; OLIVEIRA, 1999). No Brasil os frutos do grupo Santa Cruz são colhidos quando apresentam mais de 30\% da superfície em coloração rósea e vermelha que resulta num produto final de boa qualidade (MOURA; SARGENT; OLIVEIRA, 1999). Atributos como cor, tamanho, forma e defeitos externos do fruto determinam a escolha do consumidor. Entretanto estes parâmetros sozinhos não garantem a qualidade do aroma, sabor e textura do fruto (AZODANLOU et al., 2003).

A análise descritiva quantitativa (STONE et al., 1974; ABNT, 1998) é uma metodologia de avaliação sensorial que permite descrever os atributos do produto de acordo com 
interpretação dos provadores e consumidores. A avaliação do tomate de mesa convencional pela análise descritiva quantitativa (ADQ) foi empregada por alguns pesquisadores. AUERSWALD et al. (1999a) avaliaram o tempo de estocagem em tomates colhidos vermelhos durante 14 dias pela ADQ de 57 atributos. AUERSWALD et al. (1999b) compararam o crescimento de tomates longa vida hidropônicos em diferentes meios de cultivo pela ADQ de 58 atributos. FERREIRA et al. (2003) desenvolveram a terminologia descritiva para tomate de mesa através da análise descritiva quantitativa - ADQ com 18 descritores. BORGUINI (2002) encontrou diferença significativa em nível de 5\% de significância nos atributos sabor e aspecto geral quando submeteu amostras de tomate cv. Débora e Carmen cultivadas nos sistemas convencional e orgânico à análise sensorial pela escala hedônica. Porém, a análise sensorial do tomate de mesa cultivado no sistema orgânico, através da $A D Q$ não foi relatada na literatura. Em razão de escassas informações sobre análise sensorial do tomate de mesa (Lycopersicon esculentum Mill.) cultivado no sistema orgânico, este trabalho teve como objetivo analisar amostras de tomate de mesa cultivado no sistema orgânico utilizando a metodologia da análise descritiva quantitativa (ADQ).

\section{MATERIAL E MÉTODOS}

\subsection{AMOSTRAS}

Foram avaliadas quatro amostras de tomate de mesa, Lycopersicon esculentum Mill., grupo oblongo, Santa Cruz cv. Santa Clara, bilocular, cultivadas no sistema orgânico pela Associação d' Agricultura Orgânica do Paraná - AOPA e Associação de Produtores Agrícolas de Colombo - APAC. A identificação das cultivares, procedência, temperatura ambiente no dia da coleta e código das amostras analisadas estão referenciadas no quadro 1.

\section{QUADRO 1 - TOMATE DE MESA CULTIVADO NO SISTEMA ORGÂNICO COMERCIALIZADO NA REGIÃO METROPOLITANA DE CURITIBA NO PERÍODO DE FEVEREIRO DE 2000 A JANEIRO DE 2002.}

\begin{tabular}{|c|c|c|c|c|}
\hline AMOSTRA & SUBGRUPO & PROCEDÊNCIA & DATA & TEMPERATURA MÉDIA ( $\left.{ }^{\circ} \mathrm{C}\right)$ \\
\hline $\mathrm{SOl}$ & Vermelho & Campo Magro & Fevereiro/2001² & $26 \pm 2$ \\
\hline $\mathrm{SO} 2$ & Vermelho & Campo Largo & Dezembro/2001 ${ }^{1}$ & $27 \pm 2$ \\
\hline $\mathrm{SO} 3$ & $\begin{array}{l}\text { Vermelho } \\
\text { maduro }\end{array}$ & Campo Largo & Janeiro/2002² & $28 \pm 2$ \\
\hline $\mathrm{SO} 4$ & Vermelho & Colombo & Janeiro de $2002^{2}$ & $26 \pm 2$ \\
\hline
\end{tabular}

NOTA: 'primeira florada; ${ }^{2}$ última florada

\subsection{DELINEAMENTO DA AMOSTRAGEM}

Na coleta das amostras, seguindo-se o plano de amostragem da Portaria do MAARA n 553/95 (BRASIL, 1995), em razão do número de frutos necessários para as análises, foram retiradas aleatoriamente quatro caixas do local. Após a coleta, as amostras foram transportadas ao laboratório, homogeneizadas em cubas e retirados frutos de pontos e profundidades diferentes até atingir a quantidade desejada. Posteriormente, os frutos seguindo delineamento inteiramente casualizado, constituindo um arranjo fatorial $3^{2}$ (três repetições 
em duplicata), foram higienizados, agrupados em subamostras de 10 unidades cada, acondicionados em embalagens de polietileno, etiquetadas, identificadas, fechadas e conservadas em refrigeração a uma temperatura de aproximadamente $7^{\circ} \mathrm{C}$ no prazo máximo de dois dias, intervalo este entre a coleta da amostra e a análise.

\subsection{ANÁLISE SENSORIAL}

A análise foi realizada no Laboratório de Análise Sensorial do Departamento de Nutrição, por uma equipe de 10 julgadores selecionados e treinados. A avaliação foi conduzida em cabines individuais com luz branca. A análise sensorial foi conduzida de maneira que cada julgador, na mesma sessão, avaliou as três repetições das amostras, em duplicatas, devidamente codificadas. As amostras foram servidas em temperatura ambiente em pires de cor clara, acompanhadas de água mineral, faca apropriada, guardanapo de papel, ficha dos termos descritivos e ficha de avaliação. Foi utilizado o instrumento desenvolvido por FERREIRA (2003) através da metodologia da análise descritiva quantitativa (ADQ) para análise sensorial de tomate, Lycopersicon esculentum Mill., de mesa convencional e orgânico. O questionário foi montado e acompanhado dos descritores. Para quantificar cada descritor sensorial, foi utilizada uma escala não estruturada de $9 \mathrm{~cm}$, com a descrição nos pontos extremos, mínimo e máximo. A análise sensorial das amostras de tomate envolveu os seguintes descritores sensoriais: cor, defeitos de superfície, firmeza ao toque, aparência geral externa, firmeza ao corte, resistência ao corte, grau de suculência, característica da polpa, espessura da polpa, aroma, textura oral, sabor, sabor estranho, doçura, acidez, sabor remanescente, aparência geral interna e qualidade global. Também foram definidas pelos julgadores, as diferentes formas de apresentação do tomate (inteiro ou fatiado), a seqüência dos descritores e padronização do corte. Foram entregues, em primeiro lugar, os tomates inteiros para análise dos descritores: cor, defeitos na superfície, firmeza ao toque e aparência geral externa. As amostras, fatiadas em rodelas de $0,5 \mathrm{~cm}$ a partir do pedúnculo, foram analisadas através dos descritores, firmeza ao corte e resistência ao corte. A seguir os outros descritores foram avaliados. Os resultados foram avaliados pela análise de variância (Anova), fator duplo com repetição no programa Excel. Para comparação das médias foi utilizado o teste de Tukey a fim de confirmar a diferença significativa $(p<0,05)$ entre as amostra avaliadas.

\section{RESULTADOS E DISCUSSÃO}

O resultado da análise sensorial dos descritores (tabela 1) indicou que as amostras diferiram significativamente entre si $(p £ 0,05)$ em 11 dos 18 descritores analisados. Nos descritores grau de suculência, característica da polpa, aroma, sabor, sabor remanescente, aparência geral interna e qualidade global as amostras não apresentaram diferença significativa. Os perfis sensoriais dos atributos avaliados podem ser visualizados nas figuras 1 , 2 e 3.

A amostra SO3 seguida da SO2 e SO4 apresentaram a melhor intensidade de cor, que corresponde aos estádios de maturação vermelho maduro e vermelho, considerados melhores e preferidos pelos consumidores (ZAMBRANO; MOYEJA; PACHECO, 1995; MOURA; SARGENT; OLIVEIRA, 1999). Médias maiores 8,06 para cv Carmen e 7,62 para a cv. Débora foram encontradas por BORGUINI (2002). Os menores índices de defeitos de superfície que 
são atributos depreciativos do produto (MORETTI \& SARGENT, 2003; FERREIRA; FREITAS, LAZZARI, 2003), foram apresentados pelas amostras SO4 e SO3. A aparência dos frutos deve ser lisa com pequenas cicatrizes na ponta floral (apical) e peduncular, livre de queimaduras de sol, danos por insetos, dano mecânico e podridão por mofos (SUSLOW \& CANTWELL, 2003).

O pior resultado de firmeza ao toque foi da amostra SO3, foi confirmado pelos valores da firmeza ao corte e resistência ao corte. Esses resultados podem estar relacionados ao estádio de maturação dos frutos (vermelho maduro) e às condições climáticas da colheita que foi realizada em dia ensolarado numa temperatura de $28 \pm 2{ }^{\circ} \mathrm{C}$. Várias mudanças ocorrem durante o amadurecimento do fruto, uma delas é a perda da textura firme que está atrelada à estrutura e composição da parede celular, principalmente da fração péctica, que ao se degradar provoca o amolecimento nos frutos do tomate (BARRET REINA; CHITARRA; CHITARRA, 1994; MOURA; SARGENT; OLIVEIRA, 1999). Em relação a esse atributo a amostra SO2 foi a que apresentou melhor resultado, pois frutos com média firmeza e resistência ao corte são considerados melhores (WU \& ABBOT, 2002; FERREIRA et al., 2003).

TABELA 1 - ANÁLISE SENSORIAL DO TOMATE DE MESA CULTIVADO NO SISTEMA ORGÂNICO

\begin{tabular}{lcccc}
\hline \multicolumn{1}{c}{ DESCRITORES } & $\mathrm{SOl}$ & $\mathrm{SO} 2$ & $\mathrm{SO} 3$ & $\mathrm{SO} 4$ \\
\hline Cor & $4,3 \mathrm{a}$ & $6,17 \mathrm{~b}$ & $7,36 \mathrm{c}$ & $5,83 \mathrm{~b}$ \\
Defeitos de superfície & $3,22 \mathrm{a}$ & $3,71 \mathrm{a}$ & $2,55 \mathrm{ab}$ & $1,93 \mathrm{~b}$ \\
Firmeza ao toque & $4,39 \mathrm{a}$ & $5,89 \mathrm{~b}$ & $2,86 \mathrm{c}$ & $4,06 \mathrm{ab}$ \\
Aparência geral externa & $4,60 \mathrm{a}$ & $5,16 \mathrm{ab}$ & $6,00 \mathrm{bc}$ & $6,69 \mathrm{c}$ \\
Firmeza ao corte & $4,74 \mathrm{ac}$ & $5,22 \mathrm{a}$ & $3,21 \mathrm{~b}$ & $4,06 \mathrm{c}$ \\
Resistência ao corte & $4,70 \mathrm{ac}$ & $5,33 \mathrm{a}$ & $3,27 \mathrm{ba}$ & $4,12 \mathrm{ca}$ \\
Grau de suculência & $3,81 \mathrm{a}$ & $3,58 \mathrm{a}$ & $3,84 \mathrm{a}$ & $3,62 \mathrm{a}$ \\
Característica da polpa & $4,64 \mathrm{a}$ & $4,68 \mathrm{a}$ & $4,31 \mathrm{a}$ & $3,76 \mathrm{a}$ \\
Espessura da polpa & $3,42 \mathrm{ac}$ & $5,22 \mathrm{~b}$ & $4,32 \mathrm{ab}$ & $3,10 \mathrm{c}$ \\
Aroma & $6,70 \mathrm{a}$ & $6,93 \mathrm{a}$ & $6,78 \mathrm{a}$ & $6,34 \mathrm{a}$ \\
Textura oral & $3,63 \mathrm{a}$ & $3,48 \mathrm{a}$ & $2,21 \mathrm{~b}$ & $3,64 \mathrm{a}$ \\
Sabor & $4,57 \mathrm{a}$ & $4,83 \mathrm{a}$ & $4,92 \mathrm{a}$ & $4,04 \mathrm{a}$ \\
Sabor estranho & $1,51 \mathrm{a}$ & $2,61 \mathrm{bc}$ & $2,29 \mathrm{ac}$ & $2,52 \mathrm{c}$ \\
Doçura & $2,72 \mathrm{a}$ & $3,74 \mathrm{~b}$ & $4,98 \mathrm{c}$ & $3,23 \mathrm{ab}$ \\
Acidez & $3,39 \mathrm{a}$ & $2,91 \mathrm{ab}$ & $2,31 \mathrm{bc}$ & $2,84 \mathrm{ac}$ \\
Sabor remanescente & $3,40 \mathrm{a}$ & $2,81 \mathrm{a}$ & $3,28 \mathrm{a}$ & $3,08 \mathrm{a}$ \\
Aparência geral interna & $4,54 \mathrm{a}$ & $5,23 \mathrm{a}$ & $5,36 \mathrm{a}$ & $5,67 \mathrm{a}$ \\
Qualidade global & $5,94 \mathrm{a}$ & $5,75 \mathrm{a}$ & $5,71 \mathrm{a}$ & $5,85 \mathrm{a}$ \\
\hline
\end{tabular}

NOTA: médias seguidas de letras iguais não diferem entre si pelo teste de Tukey $(\mathrm{p} £$ 0,05).

Os valores da intensidade do grau de suculência encontrados pelos provadores indicaram índices próximos ao ideal para tomate. A característica da polpa que foi medida pelo aspecto liso ou esponjoso não foi encontrada diferença significativa entre as amostras avaliadas. Os resultados indicaram que a polpa dos frutos possui um aspecto próximo ao liso, considerado ideal pelos julgadores. A espessura da polpa, que pode variar de fina a grossa, apresentou na amostra SO2 valores medianos. 


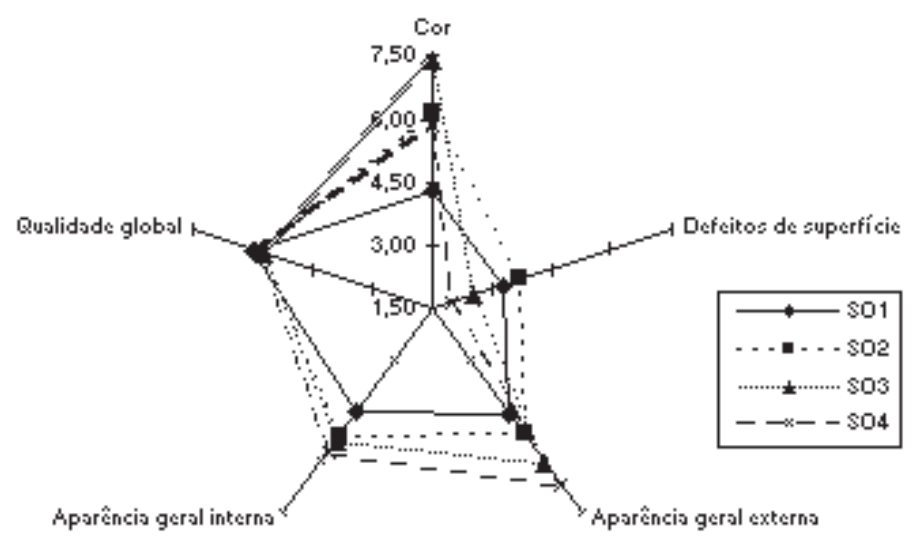

FIGURA 1 - COR, DEFEITOS DE SUPERFÍCIE, APARÊNCIA GERAL EXTERNA, APARÊNCIA GERAL INTERNA E QUALIDADE GLOBAL DO TOMATE DE MESA CULTIVADO NO SISTEMA ORGÂNICO

Para o atributo textura oral, as amostras apresentaram um valor um pouco abaixo da média, indicando que a força de mastigação necessária para o rompimento do produto coloca-o na faixa de macio a firme. A medida da textura é influenciada pela espessura da casca, firmeza da polpa e pela estrutura interna do fruto, ou seja, relação pericarpo/material placental (BARRET REINA; CHITARRA; CHITARRA, 1994; FERREIRA et al., 2003).

O resultado do sabor, que não foi detectada diferença entre as amostras, mostrou uma média entre os pontos não saboroso e saboroso que foram similares aos 6,67 para cv. Carmen e 6,91 para cv. Débora relatados por BORGUINI (2002) para amostras cultivadas no sistema orgânico. No atributo sabor estranho, todas amostras receberam um valor baixo, indicando sabor estranho não acentuado. Os resultados das amostras do atributo doçura apresentaram nítida correlação com a cor, aroma, sabor e acidez. Os termos descritivos, grau de suculência, aroma, sabor, sabor estranho, doçura, acidez e sabor remanescente estão diretamente relacionados com a qualidade do fruto que por sua vez estão correlacionados com as substâncias orgânicas, açúcares redutores, ácidos orgânicos e acidez (MOURA; SARGENT; OLIVEIRA, 1999; MAUL et al., 2000) resultantes do diferentes genótipos da cultivar, tipo de solo, condições climáticas, manejo e processos transpiratórios e respiratórios (BHOWMIK \& PAN, 1992; ZAMBRANO; MOYEJA; PACHECO, 1996; KLUGE \& MINAMI, 1997; SILVA \& GIORDANO, 2000). BORGUINI (2002) encontrou no aroma valores similares de 7,30 para cV. Carmen e 7,68 para cv. Débora.

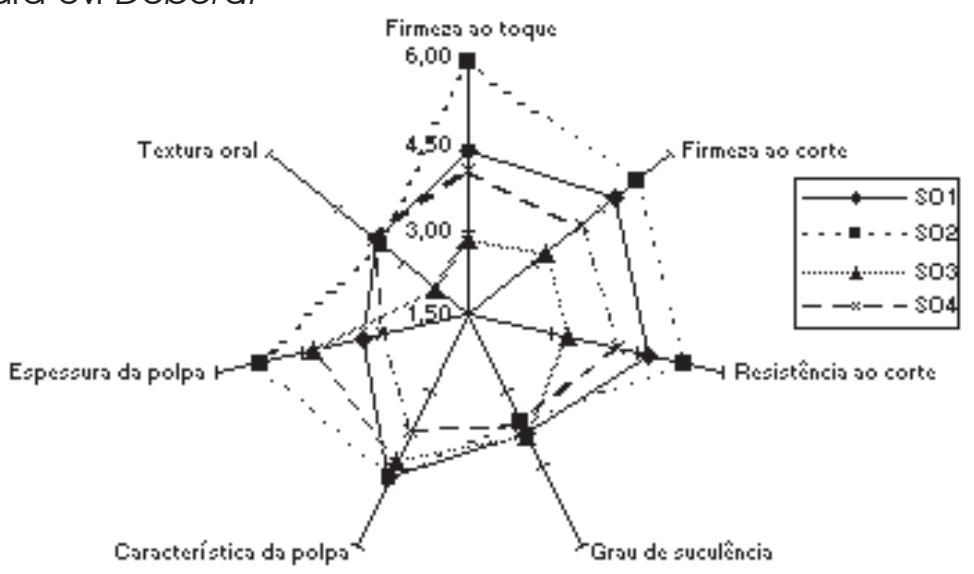

FIGURA 2 - FIRMEZA AO TOQUE, FIRMEZA AO CORTE, RESISTÊNCIA AOCORTE, SUCULÊNCIA, CARACTEÍSTICA DAPOLPA E ESPESSURA DA POLPA E TEXTURA ORAL DO TOMATE DE MESA CULTIVADO NO SISTEMA ORGÂNICO 
Os resultados do sabor remanescente, apesar de não revelarem diferenças significativas nas amostras, confirmaram uma sensação olfativa de fraca a média intensidade. Na aparência geral interna, as amostras também não apresentaram diferença significativa e confirmaram os resultados da aparência geral externa. No atributo qualidade global que não foi detectada nenhuma diferença, as amostras SOl e SO4 tenderam a melhores resultados, porém inferiores aos valores aferidos pelos julgadores nas cv. Débora $(8,06)$ e cv. Carmen $(7,62)$ cultivadas no sistema orgânico registradas por BORGUINI $(2002)$. No entanto, em relação à quase todos os atributos essas amostras (SO1 e SO4) apresentaram os piores resultados. Quando foram analisados os atributos em conjunto, isto é, todos os aspectos positivos e negativos do fruto, como aparência, cor, textura, aroma e sabor (MOURA; SARGENT; OLIVEIRA, 1999; MAUL et al., 2000, FERREIRA et al. 2003), a amostra SO2 seguida da SO3, ambas de procedência de Campo Largo (quadro 1) foram consideradas melhores. A amostra SO2, da primeira florada (quadro 1) se destacou pelo melhor aroma, sabor, baixa acidez e menor sabor remanescente.

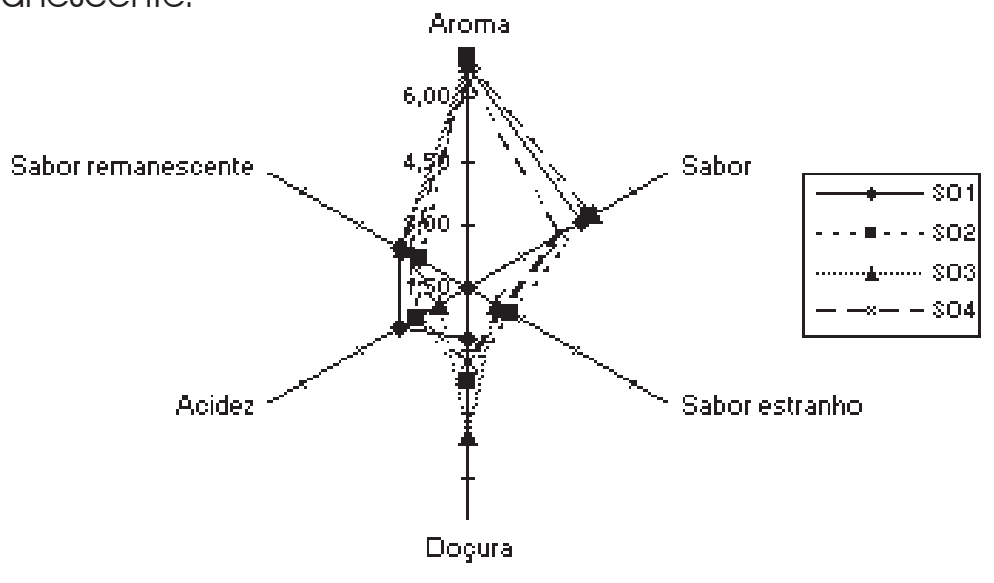

FIGURA 3 - SABOR, AROMA, SABOR ESTRANHO, DOÇURA, ACIDEZ E SABOR REMANESCENTE DO TOMATE DE MESA CULTIVADO NO SISTEMA ORGÂNICO

\section{CONCLUSÃO}

A comparação realizada pelo teste de Tukey revelou que nos descritores grau de suculência, característica da polpa, aroma, sabor, sabor remanescente, aparência geral interna e qualidade global as amostras não diferiram entre si. As amostras SO2 e SO3 de Campo Largo se destacaram pelos melhores resultados. A Amostra SO2 da primeira florada foi a preferida pelos julgadores.

\section{REFERÊNCIAS}

AUERSWALD, H.; PETERS, P.; BRÜCKNER B.; KRUMBEIN A.; KUCHENBUCH, R. Sensory analysis and instrumental measurements of short-term stored tomatoes (Lycopersicon esculentum Mill.) Postharvest Biology and Tecnology, v. 15, p. 323-334, 1999a.

AUERSWALD, H.; SCHWARZ, D.; KORNELSON, C.; KRUMBEIN A.; BRÜCKNER B. Sensory analysis, sugar and acid content of tomato at different EC values of the nutrient solution. Scientia horticulturae, v. 82, p. $227-242,1999 \mathrm{~b}$.

AZODANLOU, R.; DARBELLAY, C.; LUISIER, J.; VILLETTAZ, J.; AMADÒ, R. Development of a model for quality assessment of tomatoes and apricots. Lebensm.-Wiss. U.-Technol. v. 36, p. 223-233, 2003.

BARRET REINA, L. del C.; CHITARRA, M. I. CHITARRA, A. B. Choque a frio a atmosfera modificada no aumento de vida pós-colheita de tomates: coloração e textura. Ciênc. Tecnol. Aliment. Campinas, v. 14, n. 1, p. 14-126, jan./jun. 1994. 
BHOWMIK, S. R. \& PAN, J. C. Shelf life of mature green tomatoes stored in controlled atmosphere and high humidity. Journal of Food Science, v. 57, n. 4, p. 948-953, 1992.

BORGUINI, R. G. Tomate (Lycopersicon esculentum Mill.) orgânico: o conteúdo nutricional e a opinião do consumidor. Piracicaba, 2002. 110 f. Dissertação (Mestrado em Agronomia, área de Ciência e Tecnologia de Alimentos) - Escola Superior de Agricultura " Luiz de Queiroz".

BRASIL. Ministério da Agricultura do Abastecimento e da Reforma Agrária. Portaria $n^{\circ 0} 553$ de 30 de agosto de 1995. Dispõe sobre a Norma de Identidade, Qualidade, Acondicionamento e Embalagem do Tomate in natura, para fins de comercialização e Revoga as especificações de Identidade, Qualidade, Acondicionamento e Embalagem do Tomate, estabelecidas pela Portaria n 76, de 25 de fevereiro de 1975. Diário Oficial da República Federativa do Brasil, Brasília, set. 1995.

FERREIRA, S. M. R.; FREITAS, R. J. S.; BASSLER, T. C.; LAZZARI, E. N. Terminologia Descritiva para análise sensorial do tomate de mesa. Visão Acadêmica. Curitiba, v.4, n. 1, p.07-12, 2003.

FERREIRA, S. M. R., FREITAS, R. J. S.; LAZZARI, E. N. Defeitos do tomate de mesa (Lycopersicon esculentum Mill). Revista Higiene Alimentar. São Paulo: , v. 17, n. 1 12, p. 35-41, 2003.

KLUGE, R. A. \& MINAMI, K. Efeito de esteres de sacarose no armazenamento de tomates Santa Clara. Scientia Agrícola, Piracicaba, v. 54, n. 1-2, p. 39-44, Jan./ago. 1997.

MAUL, F.; SARGENT, S. A; SIMS, C. A.; BALDWIN, E. A.; BALABAN, M. O.; HUBER, D. J. Tomato flavor and aroma quality as affected by storage temperature. J. Food Science, v. 65, n. 7, p. 1228-1237, 2000.

MORETTI, C. L.; SARGENT, S. A. Ouro de tolo. Revista Cultivar HF, Pelotas, dez./jan. 2003.

MOURA, M. L.; SARGENT, S. A; OLIVEIRA. R. F. Efeito da atmosfera controlada na conservação de tomates colhidos em estágio intermediário de maturidade. Sci. Agric. Piracicaba, v. 56, n. 1, 1999.

SILVA, J. B. C. \& GIORDANO, L.B. Tomate para processamento industrial. Brasilia: Embrapa Comunicação para Transferência de Tecnologia - Embrapa Hortaliças, 2000. 168 p.

STONE, H. S.; SIDEL, J. L.; OLIVER. S.; WOOSLEY, A.; SINGLETON, R. C. Sensory evaluation by Quantitative Descriptive Analysis. Food Technol. v. 28, n. 11, p. 24-34, 1974.

SUSLOW T. V.; CANTWELL, M. Tomate: (Jitomate). Recomendaciones para Mantener la Calidad Postcosecha. Davis: Department of Vegetable Crops, University of California, 2003. 5p.

WU, T. \& ABBOT, J. A. Firmness and force relaxation characteristics of tomatoes stored-intact or as slices. Postharvest Biology and Tecnology, v. 24, p.60-68, 2002.

ZAMBRANO, J.; MOYEJA, J.; PACHECO, L. Efecto del estado de madurez en la composición y calidad de frutos de tomate. Agronomía tropical, v.46, n. 1, p. 61-72, 1995.

\section{Agradecimentos}

Ao Programa PIBIC/UFPR pela concessão de bolsa, Associação d'Agricultura Orgânica do Paraná - AOPA e Associação Produtores Agrícolas de Colombo - APAC que cederam as amostras. 